\title{
Using fluorescence for a full spectral analysis of dissolved and gaseous oxygen within a sand bed
}

\author{
A. Wanko, N. Forquet, R. Mose \& A.-G. Sadowski \\ Laboratoire des Systèmes Hydrauliques Urbains, \\ Université Louis Pasteur (ULP), France
}

\begin{abstract}
The mechanisms of gas transfer within a sand bed continuously and discontinuously fed were studied. Use of fluorescence technology allows us to detect both gaseous and dissolved oxygen. Magnitudes of convection and diffusion phenomena and their relative contributions to oxygen renewal of the porous medium were determined. In a continuous clear water supply, we measured gaseous oxygen concentrations of approximately $21 \%$ on the surface and $10 \%$ in-depth. These measurements are in agreement with literature values. Oxygen transfer from the gaseous phase towards the liquid phase was observed to occur entirely during the feeding phase. Thus, convection seems to be the prevalent phenomenon. In a continuous deoxygenised water supply, the gist of the gas/liquid transfer in-depth is due to the expelled air from the higher pores, which is present in the bed before feeding. The diffusion, which is an extremely slow phenomenon, aims at a homogenisation of the oxygen content in the higher part of the sand bed. In intermittent feeding, the air suction which occurs immediately after the total water infiltration is important for the oxygen renewal in the higher centimetres of the porous medium but remains insufficient to $13 \mathrm{~cm}$ depth. The oxygen transfer rates were calculated for different hydraulic load. They are all the more significant as the volumes injected by the batches were it. However, we observed that diffusion does not allow fresh air to reach deeper levels.
\end{abstract}

Keywords: convection, diffusion, oxygen, transfer, porous medium, infiltration, sanitation, sand bed. 


\section{Introduction}

The effectiveness of water treatment by means of vertical-flow sand beds mainly depends on proper soil aeration. In a number of systems, soil aeration is achieved by intermittent feeding, where oxygenation is supplied by convection and diffusion. That is the case of infiltration percolation. According to Platzer and Mauch [1], the mechanism of convection only operates if the upper surface of the bed has a large draining capacity and dries between two batches. Even though the strong influence of the hydraulic load on the clogging and aeration of beds is widely accepted, the impact and conclusions differ and are the subject of controversy. Platzer and Mauch [1] also believe that the transfer of oxygen by diffusion is $1 \mathrm{~g} \mathrm{O}_{2} / \mathrm{m}^{2} / \mathrm{h}$. They deduce a daily transfer of $22.5 \mathrm{~g} \mathrm{O}_{2} / \mathrm{m}^{2} / \mathrm{d}$. Besides, they also hypothesise that the volume of water going through the filter is replaced by an identical volume of air by convection. Kayser and Kunst [2] affirm that rapid feeding has a major positive effect on the transfer of oxygen in a filter, by diffusion and by convection alike, and show that the influence of the hydraulic load on the total oxygen transferred is overestimated by the approach of Platzer and Mauch [1]. Laber [3], who conducted similar experiments with hydraulic loads of $200-1200 \mathrm{~mm} / \mathrm{d}$ comes to similar conclusions. With the help of a one-dimensional multiphase transport code that can introduce the effects of water and air at the same time, Schwager and Boller [4] have mathematically evaluated the flow of oxygen due to convection and diffusion; the results were confirmed by experiments with tracer gas. They come to very interesting conclusions:

- convection is great over a short duration after the passing of a batch

- air diffusion and convection vary over time and it is difficult to provide a general image of the relative importance of each process

- the molecular diffusion of air is the prevalent process

- the draining level is continuously saturated and aeration from below is not possible.

However, that conclusion has no relation with the numerical values of the rate of transfer of oxygen in the gaseous phase in porous media and the rates of transfer of oxygen in the liquid phase. Several authors [5-8] have developed experimental protocols and used techniques to measure the oxygen content. These works have shown that the measurement techniques are generally a chemical process and the oxygen detected is either dissolved in water or in gaseous form. This article is based on the use of a new technology in the area, that of fluorescence. In a porous medium fed with deoxygenated water and drained, the presence of oxygen could be identified in its two forms. That made it possible to quantify the rate of transfer of oxygen in the gaseous phase and the coefficients of the transfer from the gaseous phase to the liquid phase. Continuous feeding experiments followed by prolonged draining and then by discontinuous feeding were conducted in order to bring out the impact of the feeding regime on the oxygen renewal in the gaseous phase. The influence of different parameters such as the feeding rate and the volume of the batches in the periodic feeding regime was then analysed. 


\section{Equipment and methods}

\subsection{Sand column}

The column was made up of a Plexiglas tube that was $82-\mathrm{cm}$ high, with an inner diameter of $38 \mathrm{~cm}$ and an infiltration range of $0.1134 \mathrm{~m}^{2}$. The bottom of the column was closed by a filter made of a transparent Plexiglas plate perforated with several holes with a 4.5 -mm diameter.

On the filter, the following materials were placed from the bottom up:

- Two layers of Enkamat matrix to stop the filter medium being carried away and plug the fluid draining holes

- One 5-cm high layer of $6 / 10 \mathrm{~mm}$ gravel

- A second 5-cm high layer of 4/6 $\mathrm{mm}$ gravel

Lastly, a 53-cm high layer of rolled $0 / 4 \mathrm{~mm}$ sand, which made up the main filtering bed. A spraying system in the form of a watering can head to distribute the fluid over the entire infiltration surface.

\subsection{Mass monitoring system}

The system was used to monitor the mass of the column over time, so that the statistical data relating to feeding and draining and drying were then correlated to the phenomena of air diffusion and convection in the porous medium. For that, a Sartorius Combics $1 \mathrm{CW} 1 \mathrm{P}$ model of electronic balance with a range of $300 \mathrm{~kg}$ $( \pm 0.010)$ was used, fitted with an external LT2B converter, series 980621, to convert the digital signal delivered by the balance via the digital link with the balance (RS 232 V24) into a 4-20 mA analogue signal. The balance had a 20$\mathrm{mm}$ LCD display.

\subsection{Oxygen detectors}

Foxy fiber optic oxygen sensors are spectrometer-coupled chemical sensors for the full spectral analysis of dissolved and gaseous oxygen pressure. They are portable low-power devices that offer reversibility, stability and high sensitivity. The thin film used in the probe tips consumes no oxygen, for continuous contact with the sample.

\subsubsection{Temperature compensation}

The temperature affects the time required for the fluorescence to decrease, its intensity (see figure 2), the frequency of collision of oxygen molecules with the fluorophore and the water solubility of oxygen. The graph below illustrates the variation of the intensity as a function of the temperature for a sample at a zero oxygen concentration.

\subsection{Oxygen transfer model}

The oxygen transfer model in the specific conditions is described by the Lewis and Whitman equation [9]: 


$$
\frac{\partial C}{\partial t}=k \cdot\left(C_{\infty}-C\right)
$$

where $\mathrm{k}$ and $\mathrm{C}_{\infty}$ are the oxygen transfer rate (which constant encompasses the transport and transfer phenomena) and the concentration at saturation of oxygen in the dissolved or gaseous form respectively.

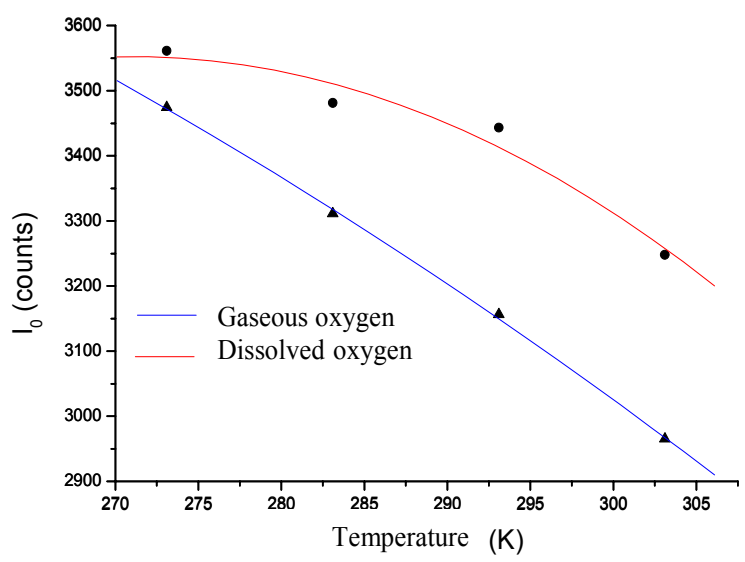

Figure 1: $\quad$ Illustration of the temperature dependence of intensity $\mathrm{I}_{0}$.

\section{Results, interpretations and discussions}

The filter was fed with clean de-oxygenated water by a sodium sulphite solution $(2 \mathrm{~g} / \mathrm{l})$. Throughout the experimentation process, the work was done with three FOXY probes arranged as follows:

- the fist one was $3 \mathrm{~cm}$ from the infiltration surface, to measure gaseous oxygen (P1)

- the second one was $13 \mathrm{~cm}$ from the infiltration surface to measure gaseous oxygen (P2)

- the third one was $43 \mathrm{~cm}$ from the infiltration surface to measure dissolved oxygen (P3)

Table 1: $\quad$ Statistical data and initial conditions (Exp_1).

\begin{tabular}{|c|c|c|}
\hline Average flow rate & & $0.04708(1 / \mathrm{s})$ \\
\hline Minimum flow rate & & $0.04194(1 / \mathrm{s})$ \\
\hline Maximum flow rate & & $0.5444(1 / \mathrm{s})$ \\
\hline Feeding time & & $455 \mathrm{~s}$ \\
\hline Injected volume & & 21.42081 \\
\hline Temperature & & $21.72^{\circ} \mathrm{C}$ \\
\hline \multirow{3}{*}{ Initial concentrations } & P1 & $10.77 \%$ \\
\hline & $\mathrm{P} 2$ & $7.38 \%$ \\
\hline & P3 & $0.39 \mathrm{mg} / \mathrm{l}$ \\
\hline
\end{tabular}




\subsection{Continuous feeding followed by draining (Exp_1)}

\subsubsection{Effect of convection}

\subsubsection{Point P1}

- Feeding phase

Observation of a convective peak after $42 \%$ of the feeding time. Oxygen transfer took place at a rate of $21.56 \% / \mathrm{min}$ (see figure 2 ).

Feeding continued and the passing of de-oxygenated water before $\mathrm{P} 1$ led to a drop in the partial pressure of gaseous oxygen (see figure 2).

- Draining phase

Once feeding had stopped, as the ponded volume above the infiltration surface seeped in, the gaseous oxygen continued to decrease (see figure 2).

After total infiltration of the ponded volume, a 3.61\% jump was observed in the oxygen, followed by accelerated re-oxygenation of the medium at a rate of $1.13 \% / \mathrm{min}$ - that rise was the result of the air suction phenomenon that immediately followed the infiltration of the ponded volume (see Figure 2). The rise completed the evidence of the short-term influence of feeding on the transfer of oxygen in the upper centimetres of the porous medium. Two phenomena were thus identified - the convection peak just at the start of the feeding phase and the suction of air after the total infiltration of the ponded volume. Diffusion was analysed later on.

\subsubsection{Point P2}

- Feeding phase

$13 \mathrm{~cm}$ away from the infiltration area, gaseous oxygen was constant during the entire feeding period (see Figure 2-right). The repercussions of the convection peak (P1) were therefore negligible, and they led to a slight rise that cannot be seen in the graph.

- Draining phase

A few moments after the feeding stopped, the partial pressure in oxygen decreased significantly, both during and after the total infiltration of the ponded volume.

The air suction observed at position P1 produced its effect at P2 11.48 minutes later, in the form of a flow of air at a speed of $0.87 \mathrm{~cm} / \mathrm{min}$, approximately the infiltration speed of the ponded water. Suction followed an exponential law, from which the rate of oxygen transfer could be deduced. $\mathrm{K}_{\mathrm{Ga}}=$ $45( \pm 7) \mathrm{h}^{-1}$.

The volume of air taken in was not sufficient for the continuation of reoxygenation of the medium at $\mathrm{P} 2$. That is why the oxygen pressure decreased again. That decrease stopped with the first effects of the diffusion of air in the medium, which occurred 44 minutes after feeding stopped (see Figure 2).

3.1.1.3 Point P3 After almost $60 \%$ of the total feeding time, no effect was observed at a depth of $43 \mathrm{~cm}$. The dissolved oxygen concentration remained equal to its initial value. 
Its increase started before the air suction phenomena observed at P1 and P2 and was therefore a consequence of the air pushed out of the pores by the incoming fluid and not immediately of the suction, which took place later (see Figure 2).

The terminal phase of rapid draining, the mixing of re-oxygenated water and de-oxygenated drained water was responsible for the decrease in oxygen at a rate of $0.1 \mathrm{mg} / \mathrm{l} . \mathrm{min}$. After long drying (nearly $17 \mathrm{~h}$ ), no consequence of the renewal of the gaseous phase was observed at P1 and P2 (see Figure 2).
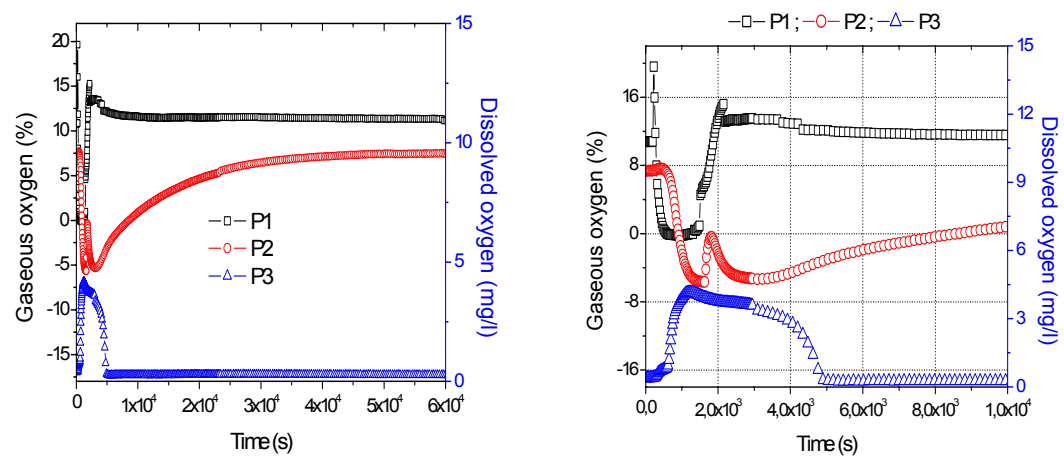

Figure 2: Change in oxygen content at the different probes (left); close-up of the effects of convection (right) (Exp_1).

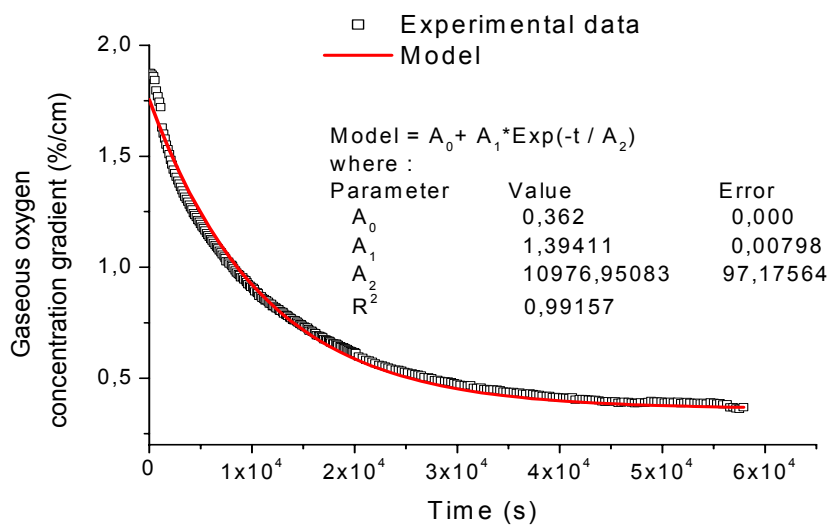

Figure 3: $\quad$ Evolution of the concentration gradient between points $\mathrm{P} 1$ and $\mathrm{P} 2$.

\subsubsection{Effect of convection}

During the long period of drying of the porous medium, a drop in the partial pressure of oxygen could be seen at P1 (3 cm deep), while it rose at P2 $(13 \mathrm{~cm}$ deep) (see Figure 2). The diffusion phenomenon therefore concerns a fairly slow homogenisation of the gaseous oxygen concentration in this part of the bed. 
Figure 3 illustrates the change in the gradient of concentration between points P1 and P2. It is seen that a significant difference of $3.62 \%$ remains in the upper 13 centimetres of the bed in spite of a fairly long rest time.

\subsection{Discontinuous or batch feeding}

The filter was fed every 10 minutes, with a volume of 7 - 11 litres per batch.

Table 2: $\quad$ Statistical data and initial conditions (Exp_2).

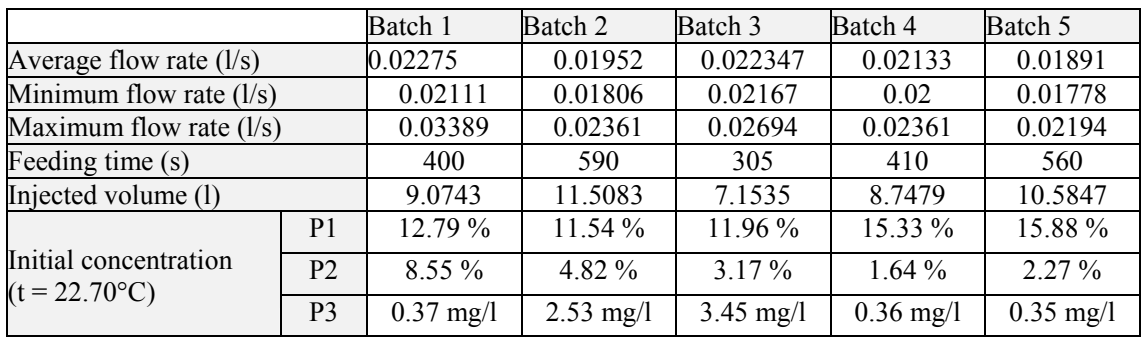

\subsubsection{Analysis of results}

\subsubsection{Point P1}

- Feeding phase

A $10.62 \%$ jump in 4 minutes (or $2.65 \%$ /minute) following the first batch was observed. That was the only convection peak during the whole experience. The initial humidification of the porous medium before a batch was therefore essential for obtaining a convection peak (see Figure 4).

Feeding with de-oxygenated water continued and the concentration dropped suddenly to $9.70 \% / \mathrm{min}$ while the feeding lasted.

- Draining phase

Feeding was stopped, and the oxygen content remained constant during the infiltration of the ponded volume.

The ponded volume was totally infiltrated and the air suction phenomenon led to an increase in the oxygen content at the rate of $1.38 \% /$ minute. The material transfer coefficients during the suction phenomena will be studied using the exponential method in the following paragraphs.

The increase in oxygen went on till a new batch was added, leading to an immediate drop. The phenomena described were reproduced periodically.

\subsubsection{Point P2}

- Feeding phase

During the entire feeding period (batch 1), the oxygen content remained constant. The peak observed $10 \mathrm{~cm}$ higher did not therefore have an impact visible at point $\mathrm{P} 2$.

- Draining phase

The passing of de-oxygenated water at point P2 led to a decrease in oxygen, which took place throughout the rapid draining period. 
Air suction at P1 after the infiltration of the ponded volume was followed by a slight increase in oxygen at position $\mathrm{P} 2$

A new decrease occurred before the arrival of the next batch. The volume taken in at P1 was therefore either not sufficient in quantity or was insufficiently diffused up to point $\mathrm{P} 2$ for re-oxygenation to continue at that point.

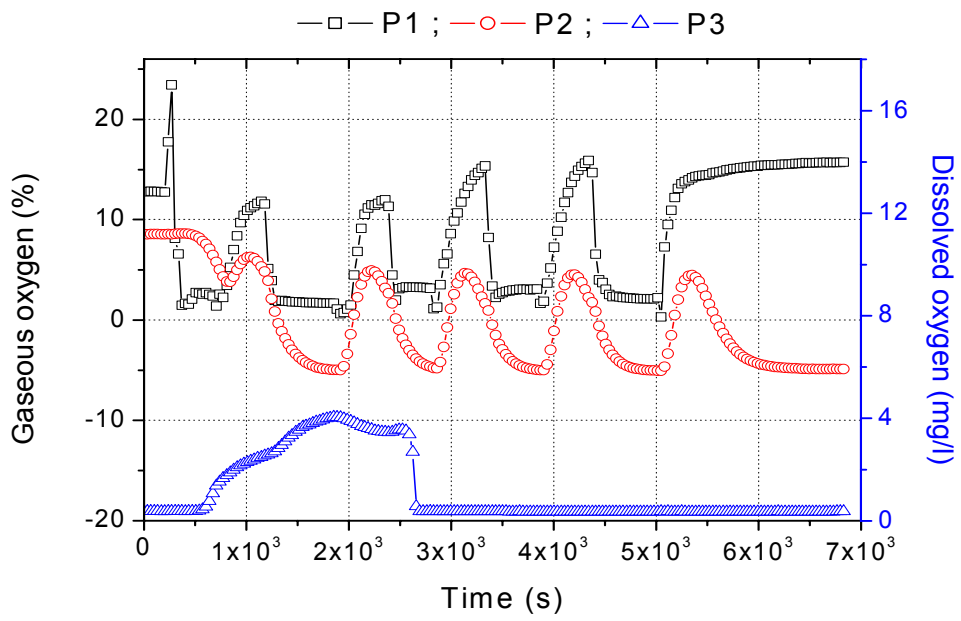

Figure 4: Changes in the oxygen content at different points (Exp_2).

3.2.1.3 Point P3 At point P3 (43 cm from the infiltration surface), the transfer of oxygen from the gaseous phase in the porous medium to the liquid phase was measured. Initially, the oxygen concentration was $0.37 \mathrm{mg} / \mathrm{l}$.

The air pushed out at the surface following the first batch led to a transfer of oxygen to the liquid phase. That led to an increase in the dissolved oxygen (see Figure 4).

The transfer process continued till the addition of the second batch, which amplified it. That phenomenon led to a new gradient on the curve.

The effects of the mixing of re-oxygenated water with the drained deoxygenated fluid led to a decrease, followed by a drop in the concentration in oxygen.

After the first two batches, the successive air suction phenomena at the surface (points P1 and P2) have no effect at point P3, where the concentration remains constantly very low.

\subsubsection{Oxygen transfer coefficients}

The table below shows the rate of transfer of oxygen in the gaseous phase $\left(K_{G} a\right)$ and the flows entering after the infiltration surface ceases to be flooded.

In the analysis of Table 2 and Table 3 a few major observations can be made: - At point P1 (3 cm deep), the highest suction rates $\left(\mathrm{K}_{\mathrm{G}} \mathrm{a}\right)$ were obtained after the infiltration of batches with larger volumes 
- At point P2 (10 cm deep), a certain harmonisation of the suction rates could be seen and the batch volumes did not therefore have any impact on reoxygenation

- The air taken in at the surface was not diffused up to point P3 (43 cm deep), the key part of the transfer was obtained by the air pushed out of the bed at the start of feeding.

From the observation of the results of this experiment, it would be fairly difficult to correlate incoming oxygen flows with the various parameters such as flow rate, feeding time or injected volume. However, the highest flow was obtained after the last batch (rest period longer, and therefore complete draining).

Table 3: $\quad$ Rate of transfer of oxygen and incoming flows (Exp_3).

\begin{tabular}{|l|c|c|c|c|c|c|c|}
\hline Phenomena & \multicolumn{2}{|c|}{ Points } & Batch 1 & Batch 2 & Batch 3 & Batch 4 & Batch 5 \\
\hline $\begin{array}{l}\text { Suction and } \\
\text { convection }\end{array}$ & $\mathrm{P} 1$ & $\mathrm{~K}_{\mathrm{G}} \mathrm{a}\left(\mathrm{min}^{-1}\right)$ & $0.39( \pm 0.05)$ & $0.71( \pm 0.06)$ & $0.26( \pm 0.01)$ & $0.25( \pm 0.02)$ & $0,58( \pm 0,04)$ \\
\cline { 2 - 8 } & $\mathrm{P} 2$ & $\mathrm{~K}_{\mathrm{G}} \mathrm{a}\left(\mathrm{min}^{-1}\right)$ & $0.4( \pm 0.1)$ & $0.32( \pm 0.07)$ & $0.38( \pm 0.08)$ & $0.34( \pm 0.08)$ & $0,39( \pm 0,05)$ \\
\hline \multicolumn{2}{|l|}{ Incoming flows } & $\mathrm{gO}_{2} / \mathrm{m}^{2}$ & 7,566 & 9.763 & 9.763 & 11.715 & 12.936 \\
\hline
\end{tabular}

\section{Conclusion}

The mechanisms of the transfer of gas in a porous medium fed continuously and discontinuously were studied. Using a new technology in the area, the authors were able to detect both gaseous and dissolved oxygen, and determine the different orders of magnitude of the phenomena of convection and diffusion and their relative importance in the re-oxygenation of a filter bed.

The fluorescence technique used by the light probes offered a number of benefits as compared to conventional electrodes. Its adaptation to different media, the non consumption of oxygen and the absence of harmful interference were some of the positive points that made the choice easier. However, the probes were found to show fairly high sensitivity if the surrounding environment was made of innumerable reflecting surfaces.

Continuous feeding led to the rarefaction of gaseous oxygen. A little while after feeding stopped, an air suction phenomenon was observed, from which the rate of transfer of gaseous oxygen could be deduced. Most of the gas/liquid transfer took place in the deeper areas as a result of the air pushed out of the upper pores. Diffusion, which is an extremely slow phenomenon, tended to homogenise the oxygen content in the upper part of the filter bed. The change in the concentration gradients within the upper $13 \mathrm{~cm}$ was a decreasing exponential function.

The importance of suction phenomena was determined for the different batches. The rates of transfer of gaseous oxygen were calculated. They were larger when the volumes injected in batches were larger. However, the diffusion 
of the volume of air taken in was insufficient, and had almost no impact at a depth. The work published [10,11] was used to extrapolate the coefficients of transfer of material in a colonised medium.

\section{References}

[1] Platzer and K. Mauch, 1997, Soil clogging in vertical flow reed beds mechanisms, parameters, consequences and.solutions? Water Science and Technology Vol 35 No 5 pp 175-181

[2] Kayser, K and Kunst, S.: 2004. Processes in Vertical-Flow reeds beds nitrification, oxygen transfer and soil clogging, $9^{\text {th }}$ international Conference on Wetland Systems for Water Pollution Control, Avignon (France) 26-30 th sept.

[3] Laber, J 2001, Bepflanzte Bodenfilter zur weitergehenden Reinigung von Oberflächenwasser und Kläranlagenabläufen, Wiener Mitteilungen, Band 167

[4] Schwager A. and Boller M., 1997, Transport Phenomena In Intermittent Filters, Wat. Sci. Tech. Vol. 35, No. 6, pp. 13-20.

[5] Koichi F., Hong-Ying H., Yasuo I. And Kohei U., 1992, Gas-liquid Oxygen Transfer Characteristics in an Aerobic Submerged Biofilter for the Wastewater Treatment Departement of Material Science and Chemical Engineering, Yokohama National University, Vol.13/14 p3745-3752.

[6] Maier B., Dietrich And Büchs J., 2001, Correct application of the sulphite oxidation methodology of measuring the volumetric mass transfer coefficient $\mathrm{K}_{\mathrm{L}} \mathrm{a}$ under non-pressurized and pressurized conditions, Institution of Chemical Engineers, Trans IchemE, Vol. 79, Part C.

[7] Amiel C., Gillot S., Roustau M. et Heduit A., 2003, Influence de la concentration en sulfite de sodium sur le transfert d'oxygène en biofiltres, Tribune de l'Eau, $\mathrm{N}^{\circ} 622 / 2$.

[8] Amiel C., Gillot S., Roustan M. Et Heduit A., 2002, Toward a Method for Measuring Oxygen Transfer in Biofilters, Water Qual. Res. J. Canada, Vol. 37, No. 4, 729-743.

[9] Lewis W.K. and Whitman W.G., 1924, Principles gas transfer absorption, Industrial and engineering chemistry, Vol.16, N. 12 , p1215-1237

[10] Boumansour B. E. and Vasel J. L., 1996, Facteur d'accroissement du transfert d'oxygène en présence de biomasse. Journal d'ETUDE DU CEBEDEAU Tribune de l'eau $\mathrm{N}^{\circ}$ 5-6/96 Sept/Oct- Nov/Déc. FUL Avenue de Longwy, 185- 6700 Arlon.

[11] Stenstrom M. K. And Gary R. G, 1980, Effects of Alpha, Beta and Theta Factor upon The Design, Specification and Operation Of Aeration Systems, Water Research, Vol. 15. pp . 643-654 